\title{
Cross-Correlations between Energy and Emissions Markets: New Evidence from Fractal and Multifractal Analysis
}

\author{
Gang-Jin Wang, ${ }^{1,2}$ Chi Xie,, ${ }^{1,2}$ Shou Chen,, and Feng Han ${ }^{3}$ \\ ${ }^{1}$ College of Business Administration, Hunan University, Changsha 410082, China \\ ${ }^{2}$ Center of Finance and Investment Management, Hunan University, Changsha 410082, China \\ ${ }^{3}$ China Merchants Bank, Shenzhen 518067, China
}

Correspondence should be addressed to Chi Xie; xiechi@hnu.edu.cn

Received 18 November 2013; Accepted 6 December 2013; Published 9 January 2014

Academic Editor: Fenghua Wen

Copyright (C) 2014 Gang-Jin Wang et al. This is an open access article distributed under the Creative Commons Attribution License, which permits unrestricted use, distribution, and reproduction in any medium, provided the original work is properly cited.

\begin{abstract}
We supply a new perspective to describe and understand the behavior of cross-correlations between energy and emissions markets. Namely, we investigate cross-correlations between oil and gas (Oil-Gas), oil and $\mathrm{CO}_{2}\left(\mathrm{Oil}-\mathrm{CO}_{2}\right)$, and gas and $\mathrm{CO}_{2}\left(\mathrm{Gas}-\mathrm{CO}_{2}\right)$ based on fractal and multifractal analysis. We focus our study on returns of the oil, gas, and $\mathrm{CO}_{2}$ during the period of April 22, 2005April 30, 2013. In the empirical analysis, by using the detrended cross-correlation analysis (DCCA) method, we find that crosscorrelations for Oil-Gas, Oil- $\mathrm{CO}_{2}$, and Gas- $\mathrm{CO}_{2}$ obey a power-law and are weakly persistent. Then, we adopt the method of DCCA cross-correlation coefficient to quantify cross-correlations between energy and emissions markets. The results show that their crosscorrelations are diverse at different time scales. Next, based on the multifractal DCCA method, we find that cross-correlated markets have the nonlinear and multifractal nature and that the multifractality strength for three cross-correlated markets is arranged in the order of Gas- $\mathrm{CO}_{2}>$ Oil-Gas $>$ Oil- $\mathrm{CO}_{2}$. Finally, by employing the rolling windows method, which can be used to investigate time-varying cross-correlation scaling exponents, we analyze short-term and long-term market dynamics and find that the recent global financial crisis has a notable influence on short-term and long-term market dynamics.
\end{abstract}

\section{Introduction}

It seems a common sense that high or low energy prices (e.g., oil and gas) are conducive to an increase or a decrease of $\mathrm{CO}_{2}$ prices [1]. For instance, Kanen [2] found that Brent crude oil prices are the main driver of natural gas prices, power prices, and $\mathrm{CO}_{2}$ prices. Alberola et al. [3] further identified that oil and gas prices are the main $\mathrm{CO}_{2}$ prices drivers by using a standard GARCH $(1,1)$ model. Fezzi and Bunn [4] investigated interrelationships among electricity, gas, and $\mathrm{CO}_{2}$ prices in the UK. They revealed that gas prices affect $\mathrm{CO}_{2}$ prices and the reaction of $\mathrm{CO}_{2}$ prices to a shock on gas prices is significant in short term. Mansanet-Bataller et al. [5] examined correlations in $\mathrm{CO}_{2}$ prices, energy, and weather. They obtained a similar conclusion that the major factors in the determination of $\mathrm{CO}_{2}$ prices are the oil and gas which are the most emission intensive energy sources. However, they also reported that extreme temperatures affect $\mathrm{CO}_{2}$ prices. This finding suggests that there are some other factors influencing $\mathrm{CO}_{2}$ prices. Ham et al. [6] studied the relationship of return volatilities between oil and $\mathrm{CO}_{2}$. They found that the relationship is complex and presents a feature of asymmetry and instability. By using models of the vector autoregressive (VAR) and the dynamic conditional correlation MGARCH (DCC-MGARCH), Chevallier et al. [1] analyzed timevarying cross-correlations in oil, gas, and $\mathrm{CO}_{2}$ prices. The results showed that cross-correlations are dynamic; for example, time-varying correlations are in the range of $[-0.05,0.05]$ between oil and $\mathrm{CO}_{2}$ and $[-0.2,0.2]$ between gas and $\mathrm{CO}_{2}$. Based on the results obtained by Chevallier et al. [1] and Ham et al. [6], we can preliminary deduce that there is not a simple linear relationship or cross-correlation between energy and emissions markets. In other words, cross-correlations in oil, gas, and $\mathrm{CO}_{2}$ prices may be nonlinear and dynamic.

The fractal and multifractal scaling behavior was widely reported in many financial time series from complex financial systems [7-9]. At the same time, many scholars confirmed that the fractal and multifractal behavior has been a "stylized 
fact" in energy markets, such as the crude oil market [10] and the electricity market [11]. The detrended fluctuation analysis (DFA), which was proposed by Peng et al. [12], is a popular fractal analysis method [13]. Kantelhardt et al. [14] extended the DFA approach to a multifractal detrended fluctuation analysis (MF-DFA) method, which can be used to analyze the multifractality in nonstationary time series. Based on the DFA method, Podobnik and Stanley [15] developed a new method in the fractal analysis to study power-law cross-correlations between two synchronized time series, which is called detrended cross-correlation analysis (DCCA). Since then, the DCCA method is widely applied to examine cross-correlations between the financial entities [16, 17]. As an example, Podobnik et al. [16] investigated 14, 981 daily observations of the Standard and Poor's 500 Index from 1950 to 2009. By using the DCCA approach, they displayed the power-law cross-correlations between price and volume volatilities. Based on the DCCA method, Wang and Xie [17] analyzed cross-correlations between Renminbi and four major currencies (i.e., USD, EUR, JPY, and KRW) in the currency basket of the Renminbi exchange rate and found that cross-correlations are weakly persistent. In order to quantify the level of cross-correlation between two synchronous time series, Zebende [18] proposed a novel detrended crosscorrelation coefficient, namely, the DCCA cross-correlation coefficient $\rho_{\mathrm{DCCA}}(s)$, which is based on the DFA and the DCCA methods. The DCCA cross-correlation coefficient is also widely used to quantify the level of cross-correlations in financial markets; for example, see [19, 20]. To detect the multifractal feature of cross-correlations between two synchronous time series, Zhou [21] extended the DCCA and MF-DFA to the method of multifractal DCCA (MF-DCCA). Besides, Kristoufek [22] further generalized the method and proposed the method of multifractal height cross-correlation analysis. Recently, the MF-DCCA approach has become a powerful technical tool to analyze the multifractality property of cross-correlations in financial markets $[23,24]$. As for the crude oil market, Wang et al. [23] employed the MF-DCCA method to study cross-correlations between the West Texas Intermediate (WTI) crude oil spot and futures return series. They found that cross-correlations are strongly multifractal for small time scales, while for large time scales crosscorrelations are nearly monofractal. In addition, Wang and Xie [24] investigated cross-correlations between the WTI crude oil market and the US stock market by using the MFDCCA method and showed that the cross-correlated behavior between the two markets is nonlinear and multifractal.

In this paper, we aim to analyze cross-correlations between energy and emissions markets from the perspective of fractal and multifractal analysis. In practical terms, we focus our attention on cross-correlations in oil, gas, and $\mathrm{CO}_{2}$ prices, namely, cross-correlations between oil and gas, oil and $\mathrm{CO}_{2}$, and gas and $\mathrm{CO}_{2}$. That is to say, as for the energy market, we choose Brent crude oil prices and Henry Hub natural gas prices as research objects. The $\mathrm{CO}_{2}$ prices are obtained from the European carbon emissions trading market that is the biggest emissions trading market at present. In the empirical analysis, to begin with, we make a preliminary analysis of the three returns of oil, gas, and $\mathrm{CO}_{2}$ in the period of April 2005-April 2013. Next, we employ the DCCA and the DCCA cross-correlation coefficient to analyze power-law cross-correlations between energy and emissions markets. Then, based on the MF-DCCA, we investigate the multifractal behavior of cross-correlations. Finally, by using the rolling windows method, we examine time-varying crosscorrelation scaling exponents, which can detect dynamics of cross-correlations.

The rest of the paper is organized as follows. We describe methodologies of the DCCA, the DCCA cross-correlation coefficient, and the MF-DCCA in Section 2. In Section 3, we show the empirical data and make a preliminary analysis. The main empirical results and analysis are presented in Section 4. Finally, we draw some conclusions in Section 5.

\section{Methodology}

2.1. DCCA Method. The DCCA method, which is used to investigate power-law cross-correlations between two different simultaneously recorded time series, was proposed by Podobnik and Stanley [15]. Supposing that there are two time series (e.g., returns) $\left\{x_{i}\right\}$ and $\left\{y_{i}\right\}$ with the equal length $N$, where $i=1,2, \ldots, N$, the DCCA method can be introduced as follows $[15,19,25]$.

Step 1. We calculate the cumulative deviation of each time series and then obtain two new sequences [19]

$$
\begin{array}{r}
X(k)=\sum_{i=1}^{k}\left(x_{i}-\bar{x}\right), \quad Y(k)=\sum_{i=1}^{k}\left(y_{i}-\bar{y}\right), \\
k=1,2, \ldots, N,
\end{array}
$$

where $\bar{x}=1 / N \sum_{i=1}^{N} x_{i}$ and $\bar{y}=1 / N \sum_{i=1}^{N} y_{i}$.

Step 2. We divide two sequences $\{X(k)\}$ and $\{Y(k)\}$ into $N_{s}$ $=\operatorname{int}(N / s)$ nonoverlapping intervals $v$. The length of each interval is $s$. Considering that the length $N$ is often not an integral multiple of the time scale $s$, a short part at the end of each sequence may be left $[14,24]$. In order not to form surplus, the same procedure is reduplicated from the opposite end of each sequence in (1). After that, we can obtain $2 N_{s}$ intervals altogether. Following Kantelhardt et al. [14] and Wang et al. [19], in our study, we set $10 \leq s \leq N / 4$.

Step 3. For each interval $v$, we define the "local trends" $\left\{\widetilde{X}_{v}(k)\right\}$ and $\left\{\widetilde{Y}_{v}(k)\right\}$ by a least-squares fit of the sequences. Then, the detrended covariance can be defined by $[14,25]$

$$
\begin{aligned}
f^{2}(v, s)=\frac{1}{s} \sum_{i=1}^{s} & \left|X[(v-1) s+i]-\widetilde{X}_{v}(i)\right| \\
\times & \left|Y[(v-1) s+i]-\widetilde{Y}_{v}(i)\right|
\end{aligned}
$$


for each interval $v, v=1,2, \ldots, N_{s}$ and

$$
\begin{aligned}
f^{2}(v, s)=\frac{1}{s} \sum_{i=1}^{s} & \left|X\left[N-\left(v-N_{s}\right) s+i\right]-\widetilde{X}_{v}(i)\right| \\
\times & \left|Y\left[N-\left(v-N_{s}\right) s+i\right]-\widetilde{Y}_{v}(i)\right|
\end{aligned}
$$

for $v=N_{s}+1, N_{s}+2, \ldots, 2 N_{s}$.

Step 4. The detrended covariance fluctuation function $F_{\text {DCCA }}^{2}(s)$ can be calculated by averaging over all intervals [24]; that is,

$$
F_{\text {DCCA }}^{2}(s)=\frac{1}{2 N_{s}} \sum_{v=1}^{2 N_{s}} f^{2}(v, s) .
$$

If the time series $\left\{x_{i}\right\}$ is identical to $\left\{y_{i}\right\}$, the DCCA method reduces to the DFA method. In practical terms, $F_{\mathrm{DCCA}}(s)$ reduces to the detrended variance $F_{\mathrm{DFA}}(s)$ described in the DFA method $[12,26]$; that is,

$$
F_{\mathrm{DFA}}(s)=\left\{\frac{1}{2 N_{s}} \sum_{v=1}^{2 N_{s}} f^{2}(v, s)\right\}^{1 / 2} .
$$

At this point, $f^{2}(v, s)=(1 / s) \sum_{i=1}^{s}\left(X[(v-1) s+i]-\widetilde{X}_{v}(i)\right)^{2}$ for each interval $v=1,2, \ldots, N_{s}$ and $f^{2}(v, s)=(1 / s) \sum_{i=1}^{s}(X[N-$ $\left.\left.\left(v-N_{s}\right) s+i\right]-\widetilde{X}_{v}(i)\right)^{2}$ for $v=N_{s}+1, N_{s}+2, \ldots, 2 N_{s}$.

Step 5. By analyzing the log-log plots of $F_{\mathrm{DCCA}}(s)$ versus $s$, we can obtain the scaling behavior of the fluctuation function [17]. If the two time series $\left\{x_{i}\right\}$ and $\left\{y_{i}\right\}$ are power-law crosscorrelated, then

$$
F_{\text {DCCA }}(s) \propto s^{\lambda}
$$

where $\lambda$ is a cross-correlation scaling exponent and is also known as an extension of Hurst exponent $H$ such that $F_{\mathrm{DFA}}(s) \propto s^{H}$ in the case of the DFA method, which can be estimated by the slope of log-log plots $F_{\text {DCCA }}(s)$ versus $s$ through ordinary least squares (OLS) [17]. Generally, the values of $\lambda$ show the type of cross-correlations between the two time series. Three cases of $\lambda$ can be summarized as follows [17] (i) If $\lambda>0.5$, the cross-correlations between the two time series are persistent (positive); namely, an increase (a decrease) in one time series is likely to be followed by an increase (a decrease) in the other time series. (ii) If $\lambda<$ 0.5 , the cross-correlations between the two time series are antipersistent (negative), which is an opposite situation to the case (i). At this point, the direction of both time series is reversed. For instance, if there is an increase of one time series, it is likely to be followed by a decrease of the other time series. (iii) When $\lambda=0.5$, the two time series are not crosscorrelated; that is, there are no correlations between the two time series [17].

2.2. DCCA Cross-Correlation Coefficient. The DCCA crosscorrelation coefficient, an extension of the DFA and the DCCA methods, was proposed by Zebende [18]. This coefficient is developed to quantify the level of cross-correlation between two synchronized time series. For each time scale $s$, the DCCA cross-correlation coefficient is defined as the ratio between the detrended covariance function $F_{\mathrm{DCCA}}^{2}(s)$ of (4) and the multiplier of two detrended variance functions $F_{\mathrm{DFA}}(s)$ of $(5)[18,20,27]$; that is,

$$
\rho_{\mathrm{DCCA}}(s)=\frac{F_{\mathrm{DCCA}}^{2}(s)}{F_{\mathrm{DFA}\left\{x_{i}\right\}}(s) F_{\mathrm{DFA}\left\{y_{i}\right\}}(s)},
$$

where $\rho_{\mathrm{DCCA}}(s)$ is a dimensionless quantity ranging from -1 to 1 [27]. At this point, it is important to note that when we calculate values of the DCCA cross-correlation coefficient for time scales $s$ in (7), the detrended covariance $f^{2}(v, s)$ in (4), that is, (2) and (3) should be calculated as $f^{2}(v, s)=(1 / s) \sum_{i=1}^{s}\left(X[(v-1) s+i]-\widetilde{X}_{v}(i)\right)(Y[(v-1) s+i]$ $\left.-\widetilde{Y}_{v}(i)\right)$ for each interval $v, v=1,2, \ldots, N_{s}$ and $f^{2}(v, s)=$ $(1 / s) \sum_{i=1}^{s}\left(X\left[N-\left(v-N_{s}\right) s+i\right]-\widetilde{X}_{v}(i)\right)(Y[N-(v$ $\left.\left.\left.-N_{s}\right) s+i\right]-\widetilde{Y}_{v}(i)\right)$ for $v=N_{s}+1, N_{s}+2, \ldots, 2 N_{s}$. For details, see [25]. Similar to the classical correlation coefficient, for each time scale $s$, a value of $\rho_{\text {DCCA }}(s)=1$ or $\rho_{\text {DCCA }}(s)=-1$ means that two series are perfectly cross-correlated or anticross-correlated, while a value of $\rho_{\mathrm{DCCA}}(s)=0$ suggests that there is no cross-correlation between two time series [17]. A prominent advantage of $\rho_{\text {DCCA }}(s)$ is that it can quantify the level of cross-correlations between two different but synchronous time series at different time scales $s$ [17].

2.3. MF-DCCA Method. Zhou [21] proposed the MF-DCCA method, which is a generalization of the DCCA method, to examine the multifractal behavior of power-law crosscorrelations between two simultaneously recorded time series. The procedure of the MF-DCCA method consists of five steps; its first three steps are identical to the DCCA procedure. Here, we only present the last two steps.

Step 4 . The $q$ th order detrended covariance fluctuation function $F_{q}(s)$ is obtained by averaging over all intervals [21, 24, 28]; that is,

$$
F_{q}(s)=\left\{\frac{1}{2 N_{s}} \sum_{v=1}^{2 N_{s}}\left[f^{2}(v, s)\right]^{q / 2}\right\}^{1 / q}
$$

for any real value $q \neq 0$ and

$$
F_{0}(s)=\exp \left(\frac{1}{4 N_{s}} \sum_{v=1}^{2 N_{s}} \ln \left[f^{2}(v, s)\right]\right)
$$

Step 5. Similar to the 5th step in the DCCA method, we can determine the scaling behavior of the fluctuation function for each $q$. If the two time series $\left\{x_{i}\right\}$ and $\left\{y_{i}\right\}$ are power-law crosscorrelated, then

$$
F_{q}(s) \propto s^{h_{x y}(q)}
$$

where $h_{x y}(q)$ is denoted as the generalized cross-correlation scaling exponent. When $q=2$, the conventional DCCA is retrieved and $h_{x y}(2)$ is equivalent to the exponent $\lambda$ in (6). 

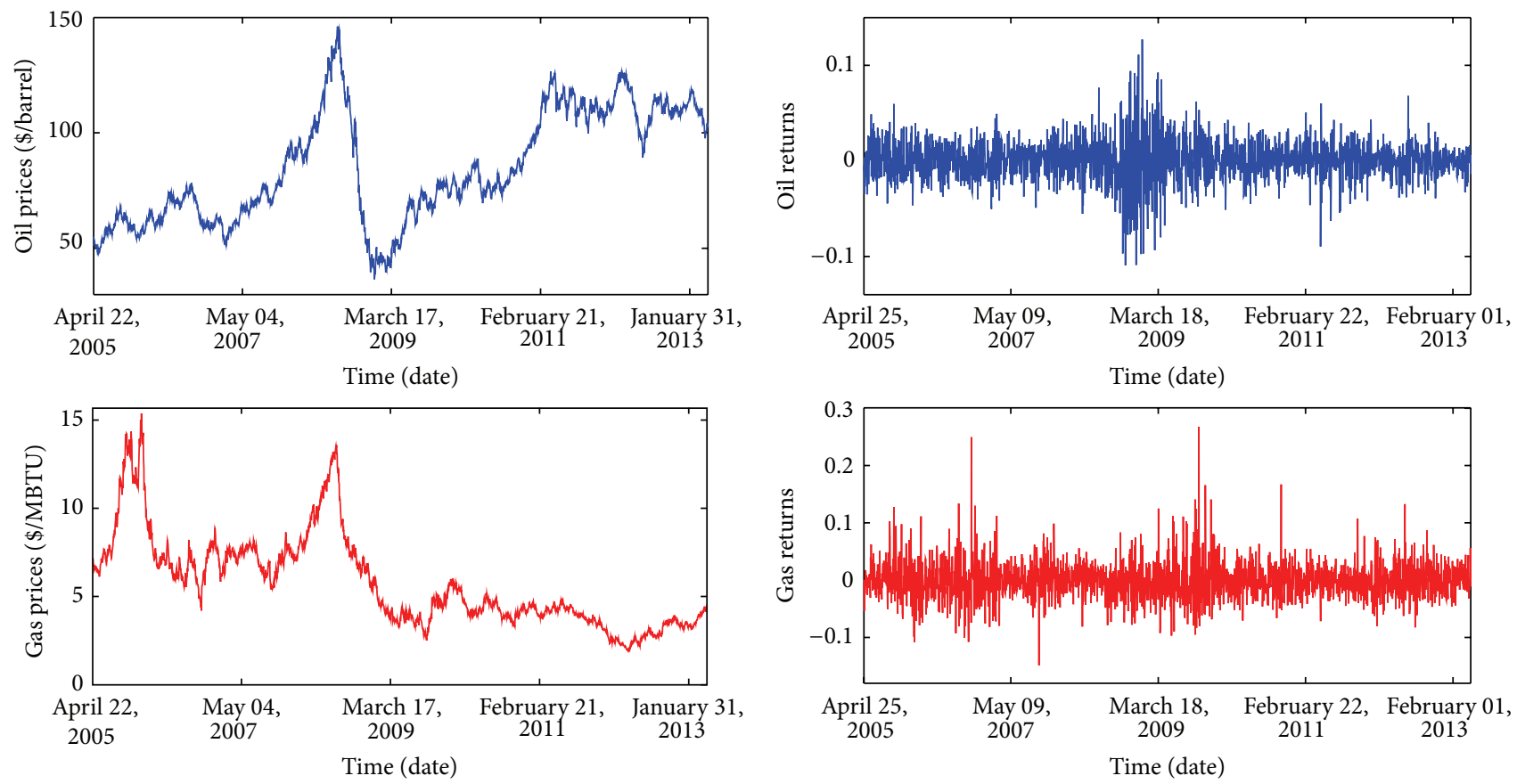

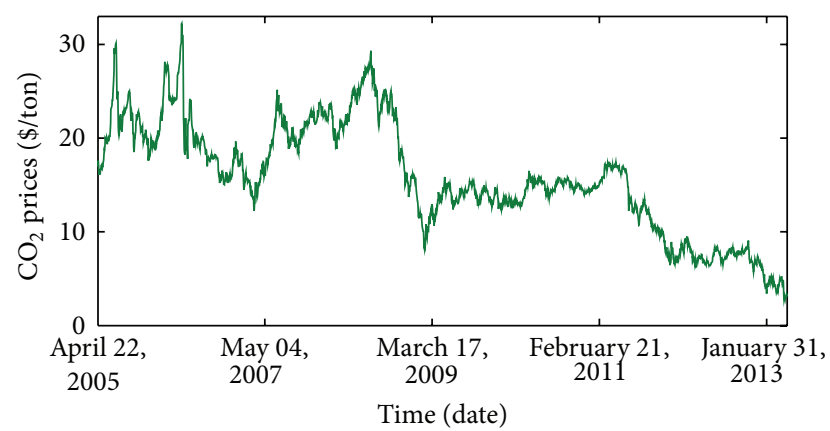

(a)

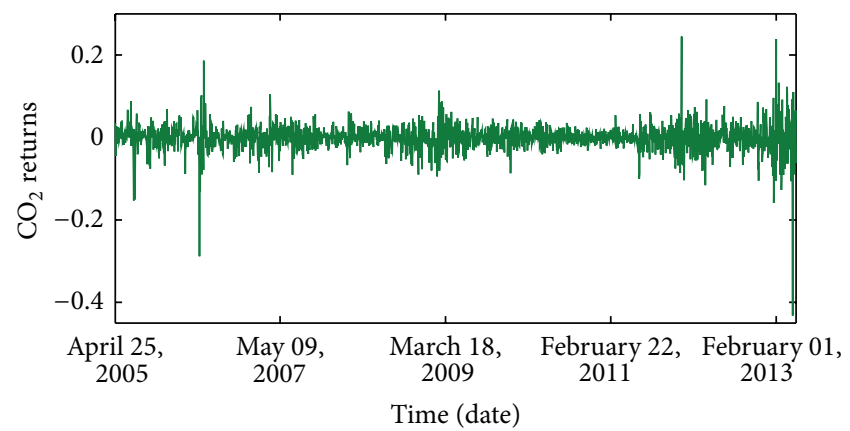

(b)

Figure 1: Prices (a) and returns (b) of oil, gas, and $\mathrm{CO}_{2}$.

TABLE 1: Descriptive statistics of returns of oil, gas, and $\mathrm{CO}_{2}$.

\begin{tabular}{lccc}
\hline & Oil & Gas & $\mathrm{CO}_{2}$ \\
\hline Mean $\left(\times 10^{-4}\right)$ & 3.0185 & -2.4506 & -8.2300 \\
Maximum & 0.1641 & 0.2677 & 0.2452 \\
Minimum & -0.1307 & -0.1489 & -0.4321 \\
Standard deviation & 0.0215 & 0.0333 & 0.0322 \\
Skewness & -0.1265 & 0.8475 & -1.2756 \\
Kurtosis & 6.6285 & 8.1827 & 26.5272 \\
Jarque-Bera $\left(\times 10^{3}\right)$ & $1.1355^{* * *}$ & $2.5521^{* * *}$ & $48.0699^{* * *}$ \\
Observations & 2060 & 2060 & 2060 \\
\hline
\end{tabular}

Notes: The Jarque-Bera statistic tests for the null hypothesis of normality in sample returns distribution. ${ }^{* * *}$ Denotes statistical significance at the $1 \%$ level.

If $x_{i}=y_{i}$ for any $i$, the MF-DCCA method is identical to the MF-DFA method [29] and $h_{x y}(q)$ reduces to $h_{x x}(q)$ or $h_{y y}(q)$ which is called the generalized autocorrelation scaling exponent (or Hurst exponent). If the value of $h_{x y}(q)$ is dependent on $q$, that is, the $h_{x y}(q)$ is a function of $q$, the crosscorrelations between the two time series are multifractal; otherwise, the cross-correlations are monofractal [24].

In order to characterize multifractality of crosscorrelations between two time series, we hereby introduce the singularity strength (or Hölder exponent) $\alpha$ and the singularity (or multifractal) spectrum $f(\alpha)$, which are defined by $[24,28]$

$$
\begin{gathered}
\alpha=h_{x y}(q)+q h_{x y}^{\prime}(q), \\
f(\alpha)=q\left[\alpha-h_{x y}(q)\right]+1,
\end{gathered}
$$

where $h_{x y}^{\prime}(q)$ represents the derivative of $h_{x y}(q)$ for $q$. Following Wang and Xie [24], we set the range of $q$ varying from -10 to 10 with a step of one. 


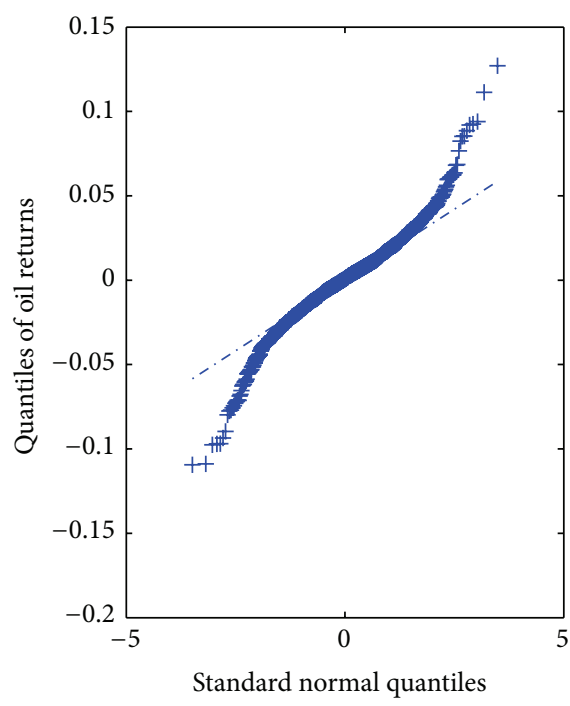

(a)

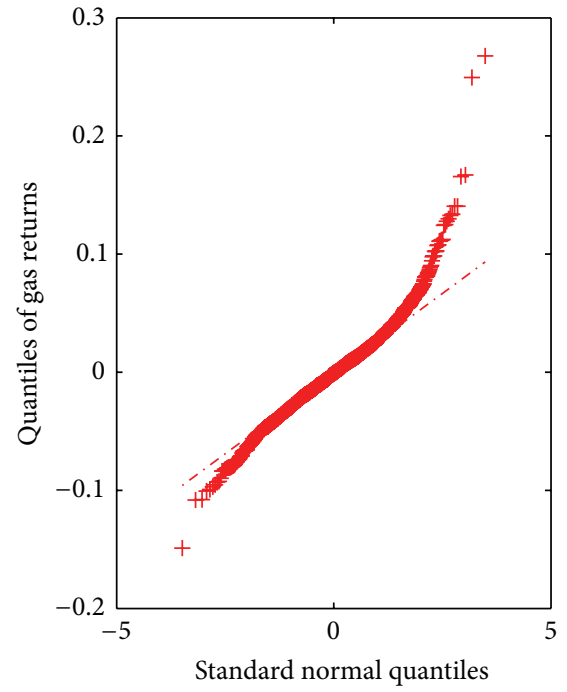

(b)

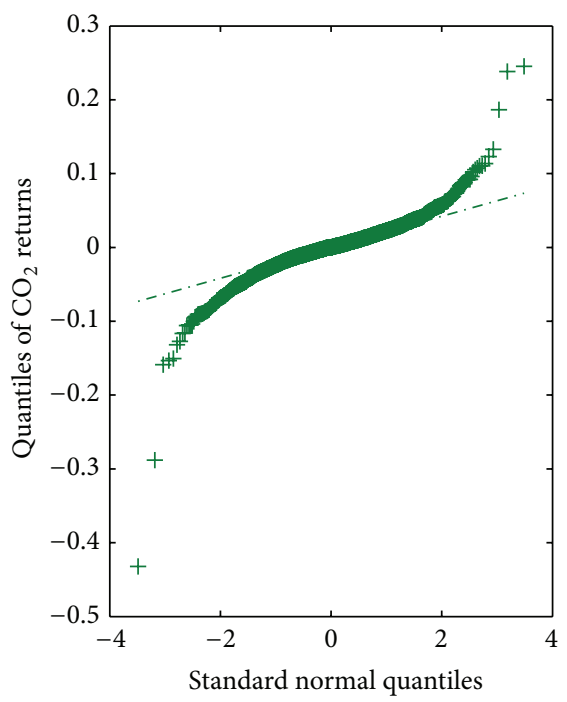

(c)

Figure 2: Normal Q-Q plots of returns of oil (a), gas (b), and $\mathrm{CO}_{2}$ (c).

\section{Data and Preliminary Analysis}

In our study, we investigate three time series of oil, gas, and $\mathrm{CO}_{2}$ daily closing prices from April 22, 2005 to April 30, 2013. Each sample includes 2061 observations. As for the oil dataset, we choose the daily Intercontinental Exchange Europe (ICE) Brent Crude Oil Futures traded in \$/barrel. The daily New York Mercantile Exchange (NYMEX) Henry Hub Natural Gas Futures traded in \$/MBTU is selected as the study object of gas. For $\mathrm{CO}_{2}$ prices, we employ the daily ICE European Climate Exchange (ECX) EU Allowance (EUA) Futures traded in $€ /$ ton. All the three futures are continuous contracts. By using the daily exchange rate obtained from the European Central Bank, we convert the unit of the prices of $\mathrm{CO}_{2}$ to $\$$. The daily closing prices of oil, gas, and $\mathrm{CO}_{2}$ are provided by the Thomson Returners Eikon.

Let $P_{t}$ denote the daily closing price on day $t$. In this paper, we focus our study on the daily logarithmic return $r_{t}$ on day $t$, which is defined as $r_{t}=\ln \left(P_{t} / P_{t-1}\right)$. Thus, each return series contains 2060 observations. The absolute return $\left|r_{t}\right|$ is called volatility. Figure 1 shows the graphical representation of prices and returns of oil, gas, and $\mathrm{CO}_{2}$. For each of the prices, as shown in Figure 1, there is a sharp decrease from the peak to the valley which occurs in the period between July 2008 and March 2009. One possible interpretation of this phenomenon is that the above-mentioned period may be the worst phrase of the global recession during the US subprime mortgage crisis. We organize descriptive statistics of the three returns in Table 1 . The mean values of the three returns are almost close to zero. The values of the standard deviation are ranged in the order of gas $>\mathrm{CO}_{2}>$ oil, which suggests that volatilities of gas and $\mathrm{CO}_{2}$ are higher than that of oil. For each return series, the Jarque-Bera statistic rejects the null hypothesis of the normal distribution at the $1 \%$ significance level. We can also find that values of skewness and kurtosis are not equal to zero and larger than three, respectively, which implies that the three returns are fat-tailed. The fat-tailed phenomena can be evidenced by results of normal Q-Q plots of the three returns in Figure 2 because each of the normal Q-Q plots is arced or " $\mathrm{S}$ " shaped.

In financial time series [30], many fat-tailed distributions have the power-law decay in the tail of the probability distribution. A lot of previous works confirmed that the "inverse cubic power-law" is found in financial markets; for instance, see [16, 23, 24, 31]. Recently, Podobnik et al. [16] proposed a new power-law estimation to investigate the fattailed distribution for financial time series. They developed an estimator of the average return interval $\tau_{\text {ave }}(q)$, which is defined as follows [24]: on average, there is one volatility above threshold $q$ after each time interval $\tau_{\text {ave }}(q)$; then

$$
\frac{1}{\tau_{\mathrm{ave}}(q)} \approx \int_{q}^{\infty} P(|x|) d|x|=P(|x|>q) \sim q^{-\beta} \text {. }
$$

The values of $\tau_{\text {ave }}(q)$ for varying $q$ can be calculated by (12) and then the estimate for $\beta$ can be obtained by the following relationship:

$$
\tau_{\text {ave }}(q) \propto q^{\beta}
$$

According to Wang and Xie [24], we set thresholds $q$ varying from $2 \sigma$ to $7 \sigma$ with a fixed step of $0.25 \sigma$, where $\sigma$ is the standard deviation of each volatility series. Then, we calculate the value of $\tau_{\text {ave }}(q)$ for each $q$ and estimate the exponent $\beta$ by (13). Finally, we display the log-log plots of $\tau_{\text {ave }}(q)$ versus threshold $q$ in Figure 3. There is a power-law relationship with Podobnik's tail exponents $\beta=3.1634, \beta=3.5185$, and $\beta=3.0735$ for oil, gas, and $\mathrm{CO}_{2}$, respectively. The three estimated Podobnik's tail exponents are close to three, which is in line with the "inverse cubic power-law" and further indicates that fat-tailed distributions widely exist in energy and emissions markets. 


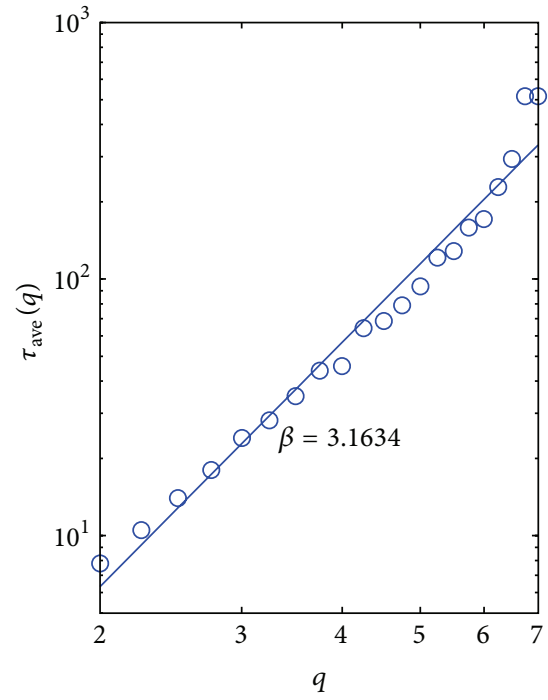

(a)

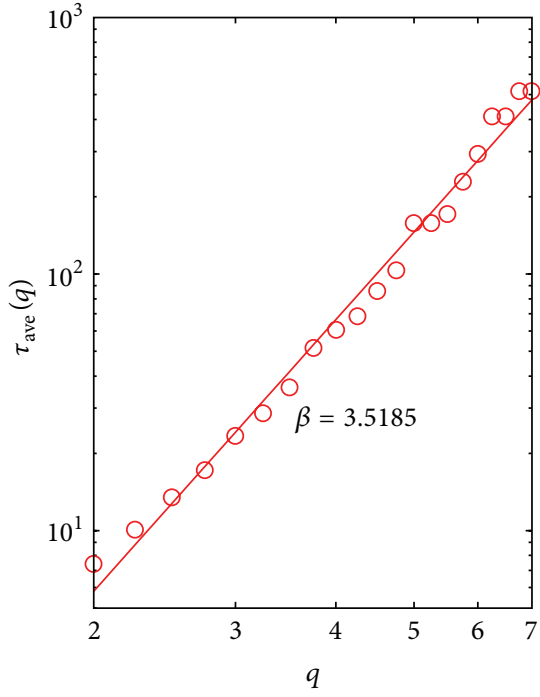

(b)

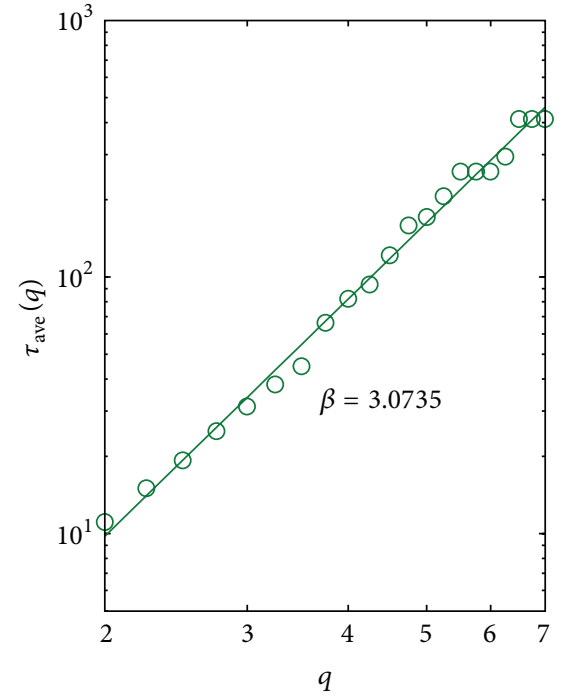

(c)

Figure 3: Log-log plots of the mean return interval $\tau_{\text {ave }}(q)$ versus threshold $q$ (in units of $\sigma$ ) for oil (a), gas (b), and $\mathrm{CO}_{2}(\mathrm{c})$.

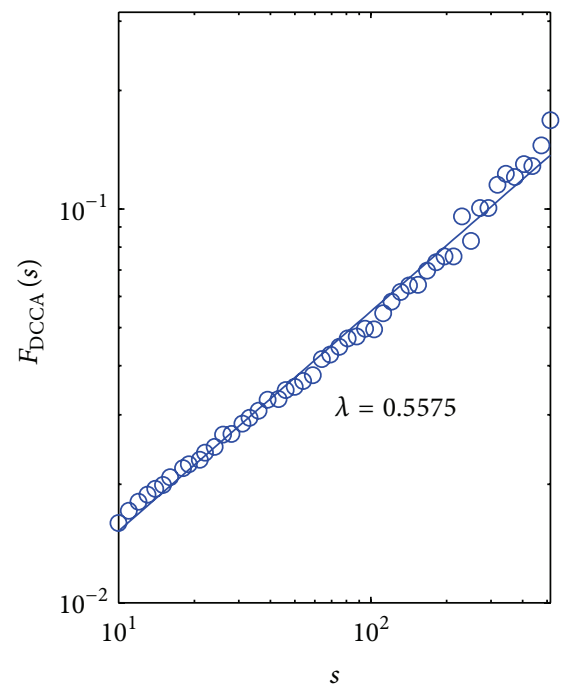

(a)

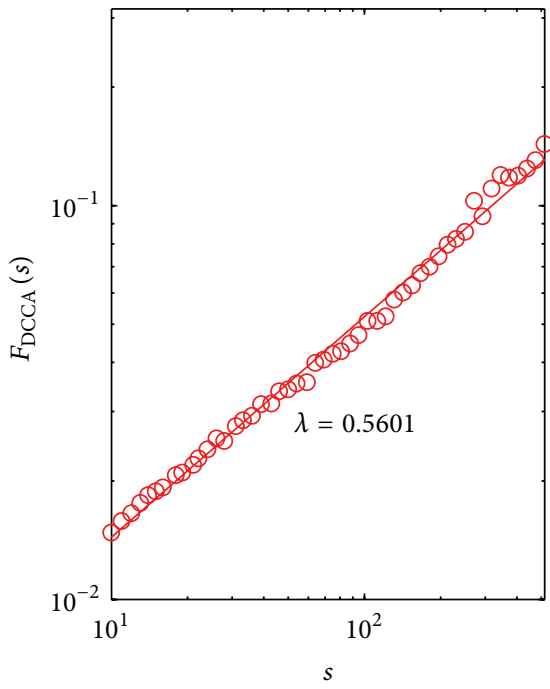

(b)

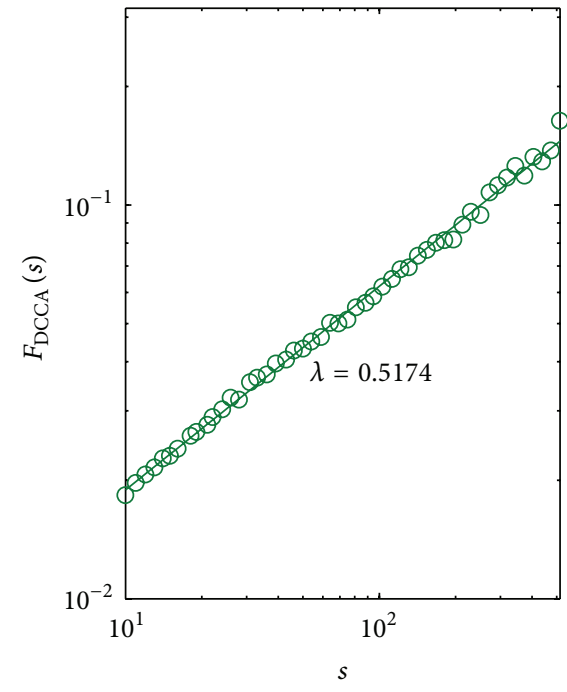

(c)

FIGURE 4: Log-log plots of the detrended covariance fluctuation function $F_{\mathrm{DCCA}}(s)$ versus time scale $s$ for oil and gas (a), oil and $\mathrm{CO}_{2}(\mathrm{~b})$, and gas and $\mathrm{CO}_{2}(\mathrm{c})$.

\section{Empirical Results and Analysis}

4.1. Cross-Correlations Analysis. Based on the DCCA method, in this subsection, we first estimate cross-correlation scaling exponents to quantitatively study cross-correlations between oil and gas, oil and $\mathrm{CO}_{2}$, and gas and $\mathrm{CO}_{2}$. For the sake of simplicity, we denote the three pairs of crosscorrelations as Oil-Gas, Oil- $\mathrm{CO}_{2}$, and Gas- $\mathrm{CO}_{2}$. The log-log plots of the detrended covariance fluctuation function $F_{\text {DCCA }}(s)$ versus time scale $s$ are drawn in Figure 4. From Figure 4, we can find that all the circle points are in a linear arrangement. Thus we can employ the OLS to estimate slopes of regression lines (see the solid lines in Figure 4), that is, cross-correlation scaling exponents, and also present the three estimated exponents in Figure 4. It can be observed that all the three cross-correlation scaling exponents are larger than 0.5 but very close to 0.5 . This finding suggests that cross-correlations between oil and gas, oil and $\mathrm{CO}_{2}$, and gas and $\mathrm{CO}_{2}$ are weakly persistent (positive). By comparing the three scaling exponents, one can see that the largest scaling exponent belongs to Oil- $\mathrm{CO}_{2}$ not Oil-Gas, which is different from the results reached by Chevallier et al. [1]. However, as suggested by Wang and Xie [17], when the compared scaling exponents are very similar, the DCCA method can only be used to analyze the type of cross-correlation which is either persistent or antipersistent. 


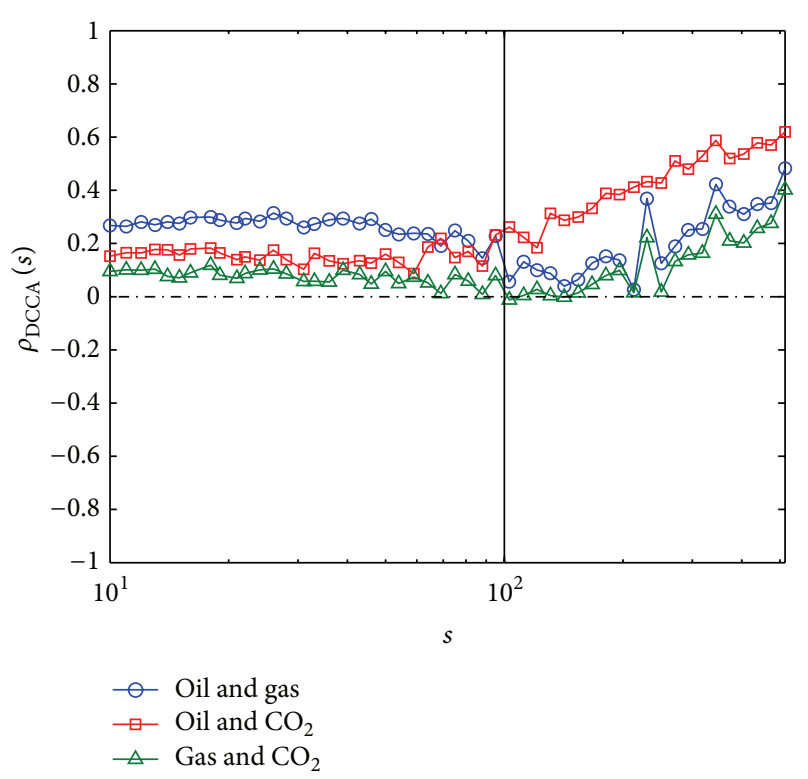

Figure 5: The DCCA cross-correlation coefficient $\rho_{\text {DCCA }}(s)$ versus time scale $s$.

Hence, we then employ the DCCA cross-correlation coefficient $\rho_{\mathrm{DCCA}}(s)$ to quantify the level of cross-correlations at different time scales. In Figure 5, we show plots of the DCCA cross-correlation coefficient $\rho_{\mathrm{DCCA}}(s)$ versus time scale $s$ for Oil-Gas, Oil- $\mathrm{CO}_{2}$, and Gas- $\mathrm{CO}_{2}$. As shown in Figure 5, one can see that DCCA cross-correlation coefficient series vary with time scales. Interestingly, when the time scale is $10<s<100$, the three coefficients for Oil-Gas, Oil$\mathrm{CO}_{2}$, and Gas- $\mathrm{CO}_{2}$ are relatively stable and are arranged in the order of Oil-Gas > Oil- $\mathrm{CO}_{2}>$ Gas- $\mathrm{CO}_{2}$. However, for $100<s<N / 4$, each coefficient presents a rising trend as time scales increase; and the order of the three coefficients is changed; that is, the position of Oil-Gas and Oil- $\mathrm{CO}_{2}$ is swapped. From the aforesaid analysis, we can obtain some inspirations as follows: (i) for Oil-Gas, Oil- $\mathrm{CO}_{2}$, and Gas- $\mathrm{CO}_{2}$, cross-correlations are diverse at different time scales, which indicates that the traditional linear correlation coefficient cannot accurately capture the diversity of crosscorrelations between energy and emissions markets; (ii) for small time scales, the strength or level of cross-correlations is arranged in the order of Oil-Gas $>$ Oil- $-\mathrm{CO}_{2}>\mathrm{Gas}-\mathrm{CO}_{2}$, which suggests that cross-correlations in the internal energy markets are stronger than those of the cross markets (i.e., cross-correlations between energy and emissions markets); and (iii) unlike case (ii), for larger time scales, the strength of cross-correlations has changed, which are arranged in the order of Oil- $\mathrm{CO}_{2}>$ Oil-Gas $>$ Gas- $\mathrm{CO}_{2}$. At this point, crosscorrelations between the cross markets (but only for Oil$\mathrm{CO}_{2}$ ) are stronger than those of the internal energy markets.

4.2. Multifractal Detrended Cross-Correlations Analysis. In this subsection, we adopt the MF-DCCA method to investigate the nonlinear and multifractal behavior of crosscorrelations between the energy and emissions markets, that

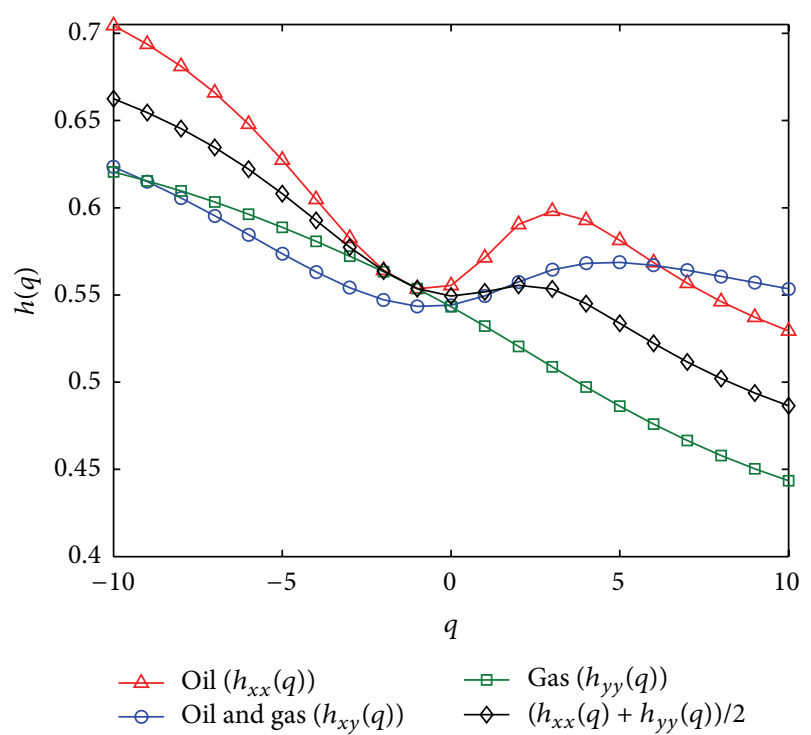

FIGURE 6: The relationship between $h(q)$ and $q$ for oil and gas.

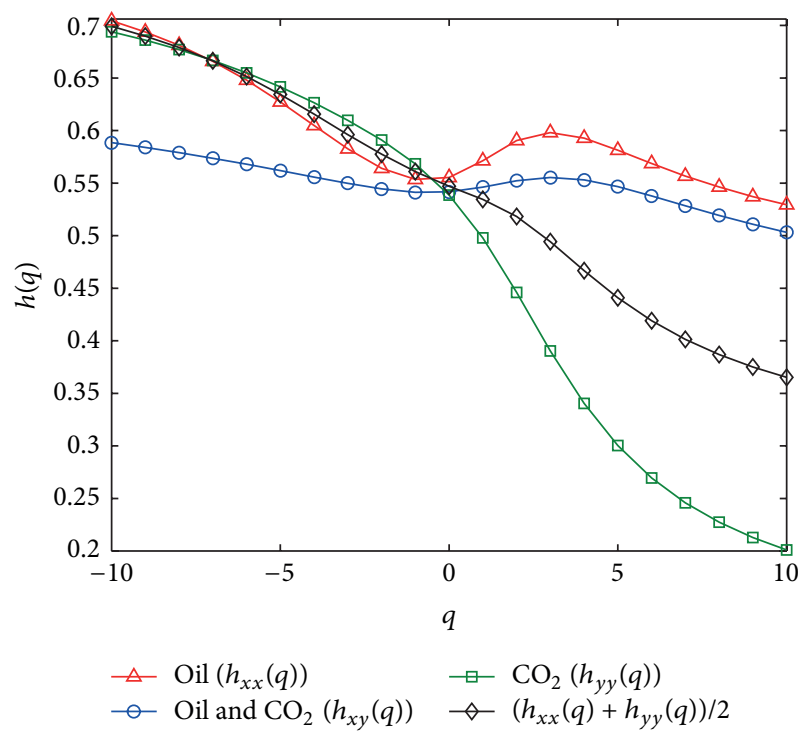

FIgURE 7: The relationship between $h(q)$ and $q$ for oil and $\mathrm{CO}_{2}$.

is, cross-correlations between oil and gas, oil and $\mathrm{CO}_{2}$, and gas and $\mathrm{CO}_{2}$. We show relationships between the crosscorrelation scaling exponent $h_{x y}(q)$ (i.e., curves with circle symbols) and $q$ for Oil-Gas, Oil- $\mathrm{CO}_{2}$, and $\mathrm{Gas}-\mathrm{CO}_{2}$ in Figures 6,7 , and 8 , respectively. At the same time, we also calculate autocorrelation scaling exponents $h_{x x}(q)$ and $h_{y y}(q)$ for the single market by the method of MF-DFA. For instance, in Figure 6, the $h_{x x}(q)$ (i.e., the curve with triangle symbols) and $h_{y y}(q)$ (i.e., the curve with box symbols) stand for autocorrelation scaling exponents for oil and gas, respectively.

According to the multifractal analysis theory, if the scaling exponent $h(q)$ is dependent on $q$, that is, the value of $h(q)$ varies with different $q$, auto-correlations or crosscorrelations are multifractal; otherwise they are monofractal [24]. As drawn in Figures 6-8, it can be found that, for 


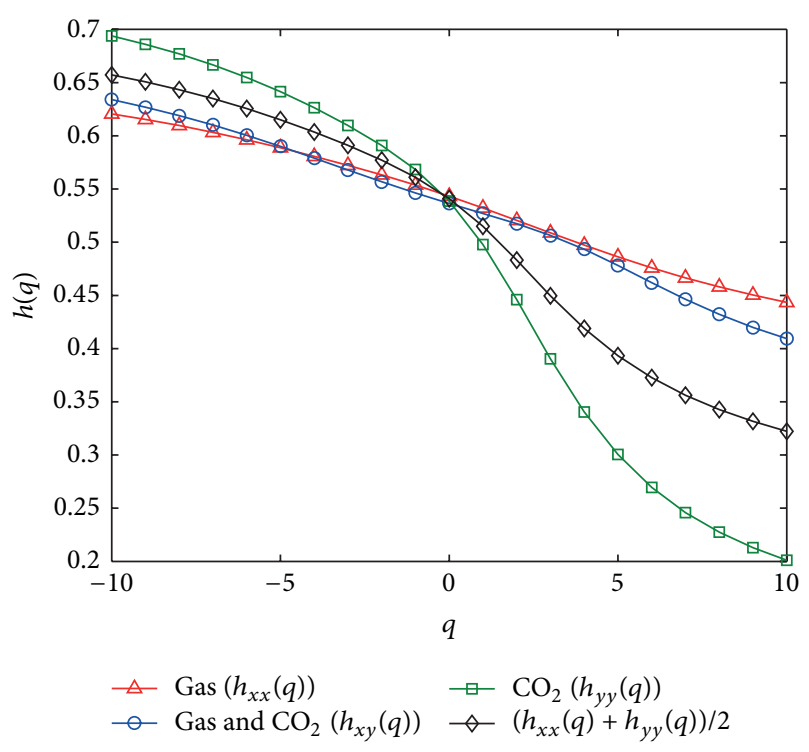

FIGURE 8: The relationship between $h(q)$ and $q$ for gas and $\mathrm{CO}_{2}$.

different $q$, the cross-correlation scaling exponent $h_{x y}(q)$ is different. That is to say, each $h_{x y}(q)$ is a nonlinear function with respect to $q$, which implies that cross-correlations between oil and gas, oil and $\mathrm{CO}_{2}$, and gas and $\mathrm{CO}_{2}$ exhibit a strong multifractal character. By analyzing the relationship between $h_{x x}(q)$ (or $\left.h_{y y}(q)\right)$ and $q$, we come to a conclusion that individual markets (i.e., gas, oil, or $\mathrm{CO}_{2}$ ) also have an evident multifractal nature.

In general, as proposed by Zhou [21], for two time series generated by a binomial measure from the $p$-model, there is a relationship among $h_{x y}(q), h_{x x}(q)$, and $h_{y y}(q)$, which is descried by

$$
h_{x y}(q)=\frac{\left(h_{x x}(q)+h_{y y}(q)\right)}{2} .
$$

Here, we denote the expression (i.e., $\left.\left(h_{x x}(q)+h_{y y}(q)\right) / 2\right)$ in the right side of (14) as the average scaling exponent. In order to verify whether the above-said equation fails or not in this empirical study, we also calculate average scaling exponents for Oil-Gas, Oil- $\mathrm{CO}_{2}$, and Gas- $\mathrm{CO}_{2}$, and show their results (i.e., the curves with diamond symbols) in Figures 6, 7, and 8, respectively. As depicted in the three figures, one can observe that, for $q<0$, the cross-correlation scaling exponent $h_{x y}(q)$ is less than the average scaling exponent $\left(h_{x x}(q)+h_{y y}(q)\right) / 2$ and greater than the average scaling exponent for $q>0$. From this, the general relationship (i.e., (14)) reported by Zhou [21] is not confirmed by our empirical result based on the analysis of energy and emissions markets. In addition, a similar result was obtained by Wang and Xie [24] who studied the crosscorrelations between the WTI crude oil market and the US stock market. This unexpected phenomenon may be due to the existence of some unknown external events and the noise trading which synchronously influence the cross-correlated behavior of the two investigated markets.

In order to better quantify the multifractality for the two markers, we further investigate the multifractal strength

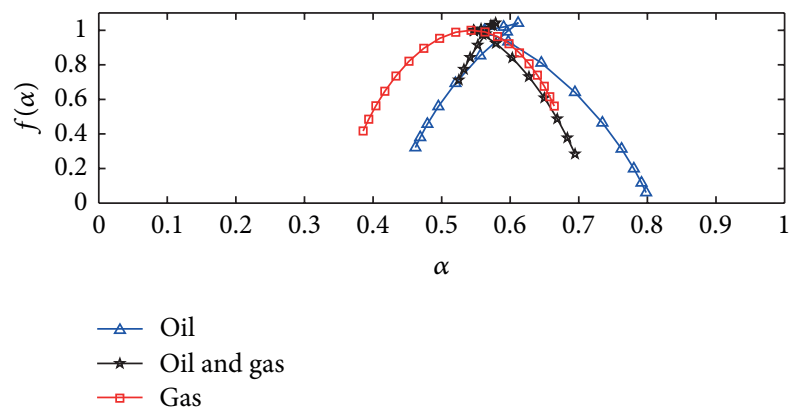

(a)

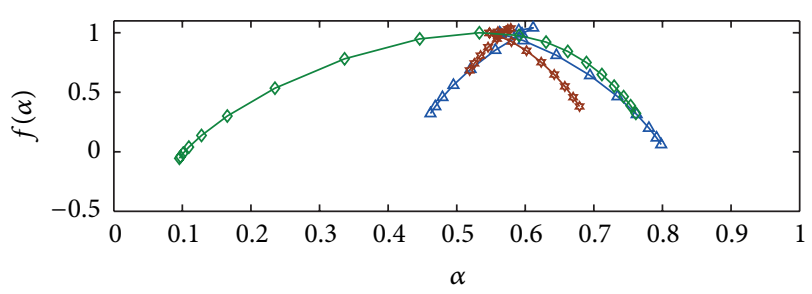

$\triangleleft$ Oil
$\nleftarrow$ Oil and $\mathrm{CO}_{2}$
$\neg \mathrm{CO}_{2}$

(b)

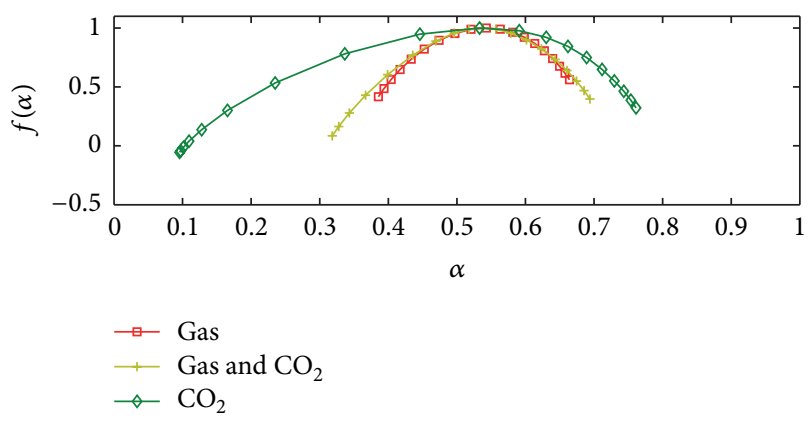

(c)

FIGURE 9: Multifractal spectra between energy and emissions markets. (a), (b), and (c) exhibit the multifractal relationships between the multifractal spectrum $f(\alpha)$ and the singularity strength $\alpha$ for oil and gas, oil and $\mathrm{CO}_{2}$, and gas and $\mathrm{CO}_{2}$, respectively. In each panel, it also shows the relationship between $f(\alpha)$ and $\alpha$ for the corresponding individual market.

by analyzing the multifractal spectra. To begin with it, based on (11), we obtain results of the multifractal spectra between the two markets and show the corresponding graphs in Figure 9. It is generally known that if the multifractal spectrum presents as a point, it is monofractal; otherwise, it is multifractal [24]. From Figure 9, we can find that all the curves for the multifractal spectra in the two markets do not show as a point. These results once again imply that the multifractality exists not only in the energy market (i.e., oil and gas) and the emissions market (i.e., $\mathrm{CO}_{2}$ ) but also in cross-correlated markets (i.e., Oil-Gas, $\mathrm{Oil}-\mathrm{CO}_{2}$, and Gas- $\mathrm{CO}_{2}$ ). Then, to examine the multifractal strength (or multifractality degree), we introduce the measure $[24,32]$

$$
\Delta \alpha=\alpha_{\max }-\alpha_{\min }
$$


TABLE 2: Multifractality degrees $\Delta \alpha$.

\begin{tabular}{lc}
\hline & $\Delta \alpha$ \\
\hline Oil & 0.3364 \\
Gas & 0.2786 \\
$\mathrm{CO}_{2}$ & 0.6657 \\
\hline Oil and gas & 0.1695 \\
Oil and $\mathrm{CO}_{2}$ & 0.1602 \\
Gas and $\mathrm{CO}_{2}$ & 0.3757 \\
\hline
\end{tabular}

where $\Delta \alpha$ stands for the width of the multifractal spectra $f(\alpha)$. The empirical results of multifractality degree are represented in Table 2. By comparing the results in Table 2 or Figure 9, we find that multifractal strengths are arranged in the order of $\mathrm{CO}_{2}>$ Oil $>$ Gas- $\mathrm{CO}_{2}>$ Gas > OilGas $>$ Oil- $\mathrm{CO}_{2}$. From this, we can draw some conclusions as follows, which may just respond to the conjectures in Section 1. (i) Whether individual markets or cross-correlated markets, they have the nonlinear and multifractal feature. Besides, except for Gas- $\mathrm{CO}_{2}$, the multifractal strengths for individual markets are larger than those of cross-correlated markets. (ii) The return series of $\mathrm{CO}_{2}$ has the largest multifractal degree, which suggests that $\mathrm{CO}_{2}$ prices have a strong multifractal feature and thus its pricing mechanism is complex and may be affected by many other external factors (e.g., weather). (iii) The multifractality exists in Gas$\mathrm{CO}_{2}$ and Oil- $\mathrm{CO}_{2}$, which indicates that an increase or a decrease of $\mathrm{CO}_{2}$ prices is not a simple feedback to a rise or a fall of the energy prices, especially gas prices. (iv) The nonlinear and multifractal behavior shows that either the separately analyzed (energy and emissions) markets or cross-correlated markets violate the random walk process; and some traditional linear bivariate models (e.g., the VAR model) may not be appropriate to detect correlations or interrelationships between the two investigated markets. So it will be an important and interesting work to develop a class of nonlinear cross-correlations models that can capture the multifractal nature [33].

4.3. Rolling Windows Analysis. To uncover the dynamic evaluation of cross-correlations between the two markets, we employ the method of rolling windows to analyze timevarying cross-correlation scaling exponents $\lambda$. The rolling windows method is also known as the local Hurst (scaling) exponent [24]. For the detailed procedure of the rolling windows method (or the local Hurst exponent), see [32].

Many scholars discussed the selection of the window size, which is a difficult issue in the rolling windows analysis because the local Hurst exponent at a given time is dependent on the window size [24]; namely, different window sizes may generate different time-varying scaling exponents. Wang and Xie [24] summarized the choice of the window size and proposed that one can choose different window sizes for different purposes. On the one hand, for a small window size such as a year, the evolution of Hurst (scaling) exponents is fierce. Thus, one can choose a small window size to examine affections of exogenous events (e.g., seasonal factors

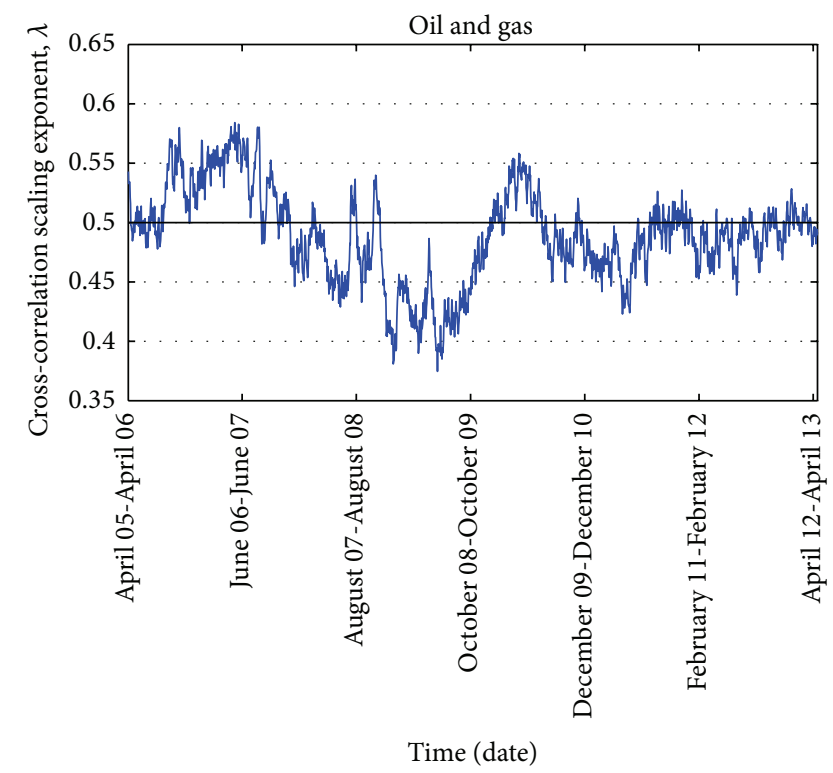

FIGURE 10: Time-varying cross-correlation scaling exponents $\lambda$ for Oil-Gas. The window size is set to 250 trading days.

and financial crisis) on short-range market dynamics. On the other hand, for a large window size, such as Tabak and Cajueiro [10] set the window size as four years, the evolution of Hurst (scaling) exponents is smooth and stable. So to investigate the major trend (e.g., market efficiency) of long-range market dynamics, one should select a large window size. In this study, we consider two window sizes (i.e., small and large window sizes) to study dynamics of crosscorrelations between the two analyzed markets. In practical terms, the small and large window sizes are fixed to 250 and 1000 trading days, respectively, which are roughly equal to one and four trading years. The step length of window is set as a single trading day for both cases. Therefore, for the window sizes of 250 and 1000 trading days, there is a total of 1811 and 1061 windows, respectively. To analyze short-term market dynamics (i.e., the window size is set to 250 trading days), we present time-varying cross-correlation exponents for Oil-Gas, Oil- $\mathrm{CO}_{2}$, and Gas- $\mathrm{CO}_{2}$ in Figures 10, 11, and 12 , respectively, while to analyze long-term market dynamics (i.e., the window size is set to 1000 trading days), we show their results in Figures 13, 14, and 15. For each figure, the time in $x$-axis represents the period of each analyzed window, that is, dates of the beginning and the last day in each analyzed window [17]. By comparing the former three figures (i.e., Figures 10-12) with the latter three ones (i.e., Figures 13$15)$, we can find that dynamics of cross-correlation scaling exponents of the latter are relatively smoother than those of the former, which is just as expected.

From Figure 10, we can observe that cross-correlation scaling exponents for Oil-Gas vary in the range of $[0.38,0.58]$. For the whole period, except for the period during the US subprime mortgage crisis, time-varying scaling exponents are in the interval $[0.45,0.55]$ and its curve (or tendency) fluctuates above and below 0.5 . These results imply that 


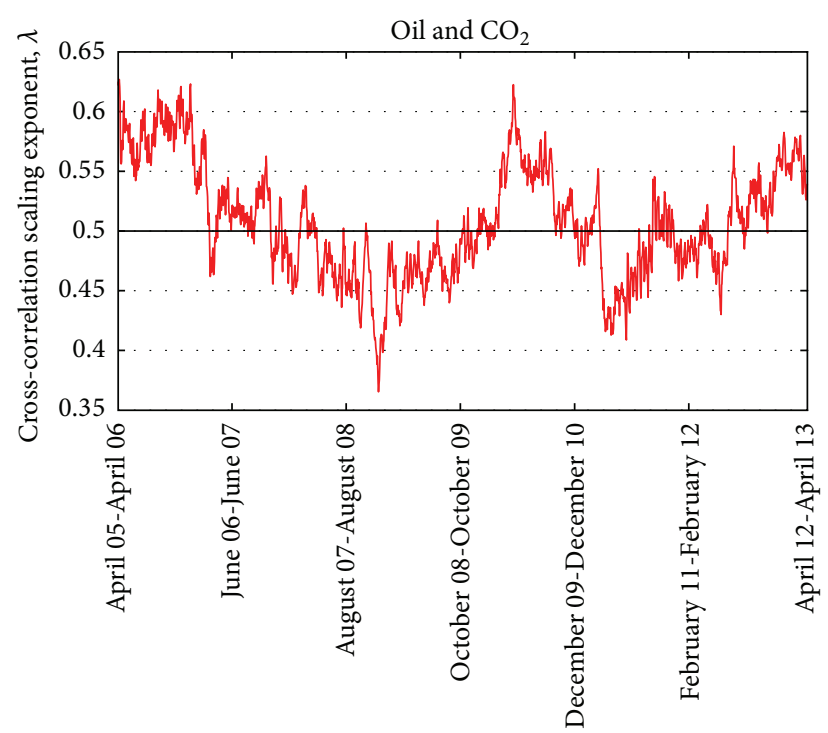

Time (date)

FIGURE 11: Time-varying cross-correlation scaling exponents $\lambda$ for Oil- $\mathrm{CO}_{2}$. The window size is set to 250 trading days.

the dynamic performance of cross-correlations between oil and gas is relatively stable, except for the impact by the 2008 financial crisis. An interesting finding is that about $1138 / 1811 \approx 61.48 \%$ of scaling exponents are less than 0.5 ; that is, more than half of cross-correlations between oil and gas are antipersistent. One possible explanation to this phenomenon is that the oil and gas are the substitute goods because an increase (a decrease) in one product's sales (prices) will reduce the potential sales of another product. As shown in Figure 11, it can be seen that crosscorrelation scaling exponents between oil and $\mathrm{CO}_{2}$ have a trend of cyclical fluctuation. The fluctuation trend shows a slow decrease firstly and then a rapid increase. Similar to Oil-Gas, during the US subprime mortgage crisis, the cross-correlation scaling exponents for Oil- $\mathrm{CO}_{2}$ exhibit a dramatic fluctuation and most of them are less than 0.5. More than half (about 53\%) of cross-correlation scaling exponents are greater than 0.5 , which suggests that Oil$\mathrm{CO}_{2}$ has a long-range positive cross-correlation. As for Gas$\mathrm{CO}_{2}$, in Figure 12, one can find that cross-correlation scaling exponents show high volatilities over time and no law can be found in them. In addition, as plotted in Figure 12, it can be observed that most of cross-correlation scaling exponents are larger than 0.5. That is, during most of the period, gas and $\mathrm{CO}_{2}$ are positively cross-correlated.

Before the period or time window, for which the beginning date is April 2008, in Figure 13, we find that the cross-correlation scaling exponents are in the range $[0.49,0.54]$ which are very close to 0.5 . This finding implies that, from a long-term point of view, the cross-correlated market (i.e., the crude oil market and natural gas market) is a weakly efficient market. However, after the US subprime mortgage crisis, exponents for Oil-Gas experience a large change, which are all smaller than 0.5 . From this, we can

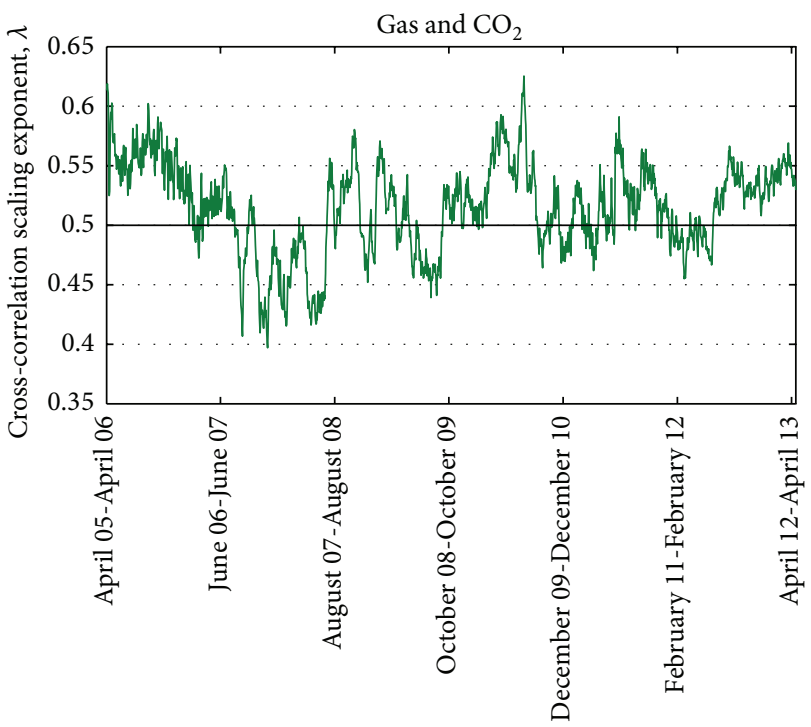

Time (date)

FIGURE 12: Time-varying cross-correlation scaling exponents $\lambda$ for Gas- $\mathrm{CO}_{2}$. The window size is set to 250 trading days.

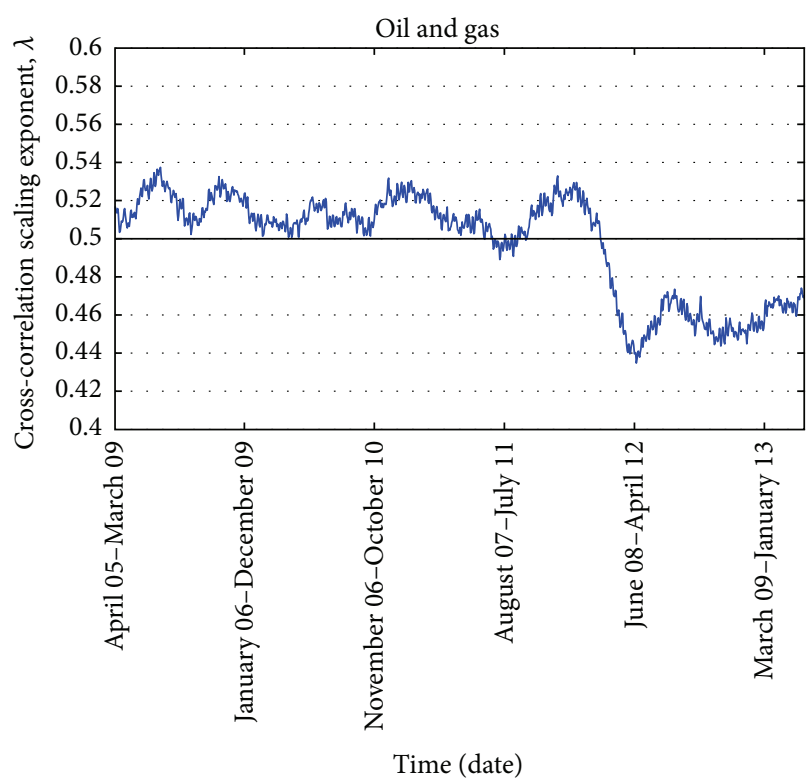

FIGURE 13: Time-varying cross-correlation scaling exponents $\lambda$ for Oil-Gas. The window size is set to 1000 trading days.

draw a conclusion that the 2008 financial crisis has a longrun influence on the cross-correlated market. Interestingly, from Figure 14, one can see that most of cross-correlation scaling exponents for Oil- $\mathrm{CO}_{2}$ are greater than 0.5 , which indicates that cross-correlations between oil and $\mathrm{CO}_{2}$ are persistent. This result confirms that crude oil prices are the main driver of $\mathrm{CO}_{2}$ prices from a long-term perspective. As shown in Figure 15, it can be observed that the trend of cross-correlation scaling exponents for Gas- $\mathrm{CO}_{2}$ is similar to that of Oil-Gas. Similar to Oil-Gas, the financial crisis has 


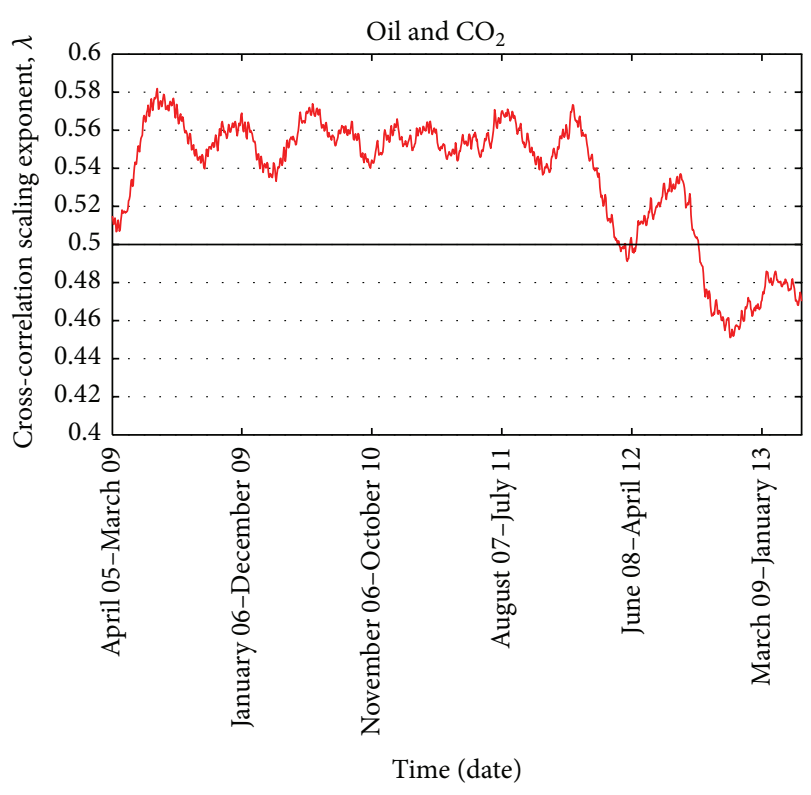

FIGURE 14: Time-varying cross-correlation scaling exponents $\lambda$ for Oil- $\mathrm{CO}_{2}$. The window size is set to 1000 trading days.

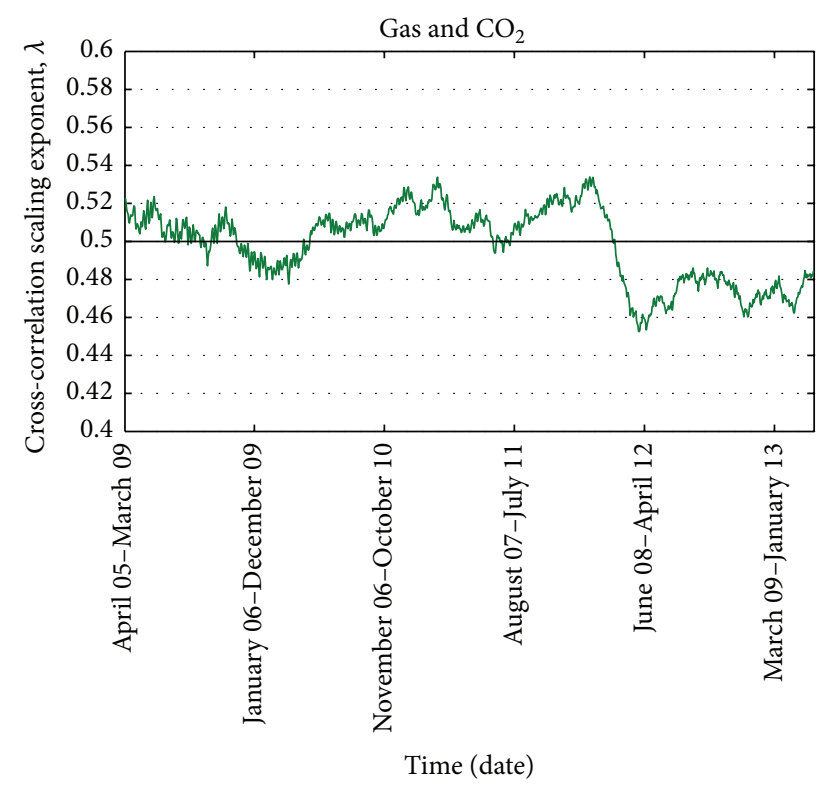

FIGURE 15: Time-varying cross-correlation scaling exponents $\lambda$ for Gas- $\mathrm{CO}_{2}$. The window size is set to 1000 trading days.

a marked impact on the Oil- $\mathrm{CO}_{2}$ and Gas- $\mathrm{CO}_{2}$ which leads to dramatic changes on cross-correlation scaling exponents.

\section{Conclusion}

In this study, we focus our study on cross-correlations between energy and emissions markets from a perspective of fractal and multifractal analysis. Namely, we take a fresh look at cross-correlations between oil and gas, oil and $\mathrm{CO}_{2}$, and gas, and $\mathrm{CO}_{2}$. We choose returns of the oil, gas and $\mathrm{CO}_{2}$ during the period of April 22, 2005-April 30, 2013 as the research sample. In the empirical process, we first use the methods of the DCCA and the DCCA cross-correlation coefficient to examine power-law cross-correlations and the level of cross-correlations, respectively. Then, we employ the MF-DCCA approach to analyze the multifractal behavior of cross-correlations and quantify multifractal strengths of individual and cross-correlated markets. Finally, by using the rolling windows method, from short-term and long-term perspectives, we investigate time-varying cross-correlation scaling exponents, which can capture dynamics of crosscorrelations.

The basic findings in our study can be summarized as follows. (i) On the basis of the analysis of descriptive statistics and the Podobnik's tail exponent, we find that the three returns of oil, gas, and $\mathrm{CO}_{2}$ are fat-tailed and obey the "inverse cubic power-law." (ii) By employing the DCCA method, we find that cross-correlations between oil and gas, oil and $\mathrm{CO}_{2}$, and gas and $\mathrm{CO}_{2}$ are weakly persistent. (iii) The cross-correlation coefficients for Oil-Gas, Oil- $\mathrm{CO}_{2}$, and Gas$\mathrm{CO}_{2}$ are different at different time scales. (iv) The nonlinear and multifractal nature is also found in individual and crosscorrelated markets. For cross-correlated markets, Gas- $\mathrm{CO}_{2}$ has the largest multifractality degree.

In addition, we investigate short-term and long-term market dynamics of cross-correlations and come to some results as follows. On the one hand, for short-term market dynamics, (i) cross-correlation scaling exponents for Oil-Gas, Oil- $\mathrm{CO}_{2}$, and Gas- $\mathrm{CO}_{2}$ show a drastic fluctuation; (ii) oil and $\mathrm{CO}_{2}$ and gas and $\mathrm{CO}_{2}$ are positively cross-correlated for most of the period; and (iii) the dynamic performance of the three cross-correlation scaling exponents is notably affected by the global financial crisis. On the other hand, for long-term market dynamics, (iv) the three cross-correlated markets are also influenced by the financial crisis; (v) for the wholly analyzed period, except for the period after the financial crisis, cross-correlated markets for Oil-Gas and Gas$\mathrm{CO}_{2}$ exhibit as a weakly efficient market. Oil- $\mathrm{CO}_{2}$ has a longrange positive cross-correlation.

Through this empirical analysis, we supply a new perspective to describe and understand cross-correlations between energy and emissions markets. From fractal and multifractal analysis, we obtain some new results, such as positive power-law cross-correlations, different DCCA crosscorrelation coefficients at different time scales, the nonlinear and multifractal cross-correlated behavior, and short-term and long-term market dynamics, which are new insights in energy and emissions markets, especially in the field of energy economics. At the same time, our results also can be added as a new factor or view to consider for banking, finance sectors, and so forth. As an extension of this study, an urgent and interesting future work is needed to design a new kind of cross-correlation models that can capture the nonlinear and multifractal nature.

\section{Conflict of Interests}

The authors declare that there is no conflict of interests regarding the publication of this paper. 


\section{Acknowledgments}

The authors thank C. Yu who works in the Guosen Securities Co., Ltd., for helpful discussions. This work was supported by the Scholarship Award for Excellent Doctoral Student granted by the Ministry of Education of China, the Hunan Provincial Innovation Foundation for Postgraduate (Grant no. CX2013A006), the National Natural Science Foundation of China (Grant no. 71373072), the Specialized Research Fund for the Doctoral Program of Higher Education (Grant no. 20130161110031), the China Postdoctoral Science Foundation (Grant no. 2013M530376), and the Foundation for Innovative Research Groups of the National Natural Science Foundation of China (Grant no. 71221001).

\section{References}

[1] J. Chevallier, "Time-varying correlations in oil, gas and $\mathrm{CO}_{2}$ prices: An application using BEKK, CCC and DCC-MGARCH models," Applied Economics, vol. 44, no. 32, pp. 4257-4274, 2012.

[2] J. L. M. Kanen, Carbon Trading and Pricing, Environmental Finance, London, UK, 2006.

[3] E. Alberola, J. Chevallier, and B. Chèze, "Price drivers and structural breaks in European carbon prices 2005-2007," Energy Policy, vol. 36, no. 2, pp. 787-797, 2008.

[4] C. Fezzi and D. W. Bunn, "Structural interactions of European carbon trading and energy prices," The Journal of Energy Markets, vol. 2, no. 4, pp. 53-69, 2009.

[5] M. Mansanet-Bataller, A. Pardo, and E. Valor, " $\mathrm{CO}_{2}$ prices, energy and weather," Energy Journal, vol. 28, no. 3, pp. 73-92, 2007.

[6] R. Ham, R. A. Ratti, and K. H. Yoon, "Oil and carbon prices: asymmetries, instability and spillovers," in Proceedings of the 41st Australian Conference of Economists, p. 119, Victoria University, Melbourne, Australia, July 2012.

[7] J. Kwapień and S. Drożdż, "Physical approach to complex systems," Physics Reports, vol. 515, no. 3-4, pp. 115-226, 2012.

[8] F. Wen, Z. Li, C. Xie, and S. David, "Study on the fractal and chaotic features of the Shanghai composite index," Fractals, vol. 20, no. 2, pp. 133-140, 2012.

[9] Z.-Q. Jiang and W.-X. Zhou, "Multifractal detrending movingaverage cross-correlation analysis," Physical Review E, vol. 84, no. 1, Article ID 016106, 11 pages, 2011.

[10] B. M. Tabak and D. O. Cajueiro, "Are the crude oil markets becoming weakly efficient over time? A test for time-varying long-range dependence in prices and volatility," Energy Economics, vol. 29, no. 1, pp. 28-36, 2007.

[11] J. Alvarez-Ramirez and R. Escarela-Perez, "Time-dependent correlations in electricity markets," Energy Economics, vol. 32, no. 2, pp. 269-277, 2010.

[12] C.-K. Peng, S. V. Buldyrev, S. Havlin, M. Simons, H. E. Stanley, and A. L. Goldberger, "Mosaic organization of DNA nucleotides," Physical Review E, vol. 49, no. 2, pp. 1685-1689, 1994.

[13] C. Chen and L. Wang, "Dual fractal dimension and long-range correlation of Chinese stock prices," Journal of the Physical Society of Japan, vol. 81, no. 3, Article ID 034801, 6 pages, 2012.

[14] J. W. Kantelhardt, S. A. Zschiegner, E. Koscielny-Bunde, S. Havlin, A. Bunde, and H. E. Stanley, "Multifractal detrended fluctuation analysis of nonstationary time series," Physica A, vol. 316, no. 1-4, pp. 87-114, 2002.

[15] B. Podobnik and H. E. Stanley, "Detrended cross-correlation analysis: a new method for analyzing two nonstationary time series," Physical Review Letters, vol. 100, no. 8, Article ID 084102, 4 pages, 2008.

[16] B. Podobnik, D. Horvatic, A. M. Petersen, and H. E. Stanley, "Cross-correlations between volume change and price change," Proceedings of the National Academy of Sciences of the United States of America, vol. 106, no. 52, pp. 22079-22084, 2009.

[17] G. -J. Wang and C. Xie, "Cross-correlations between Renminbi and four major currencies in the Renminbi currency basket," Physica A, vol. 392, no. 6, pp. 1418-1428, 2013.

[18] G. F. Zebende, "DCCA cross-correlation coefficient: quantifying level of cross-correlation," Physica A, vol. 390, no. 4, pp. 614618, 2011.

[19] G.-J. Wang, C. Xie, Y.-J. Chen, and S. Chen, "Statistical properties of the foreign exchange network at different time scales: evidence from detrended cross-correlation coefficient and minimum spanning tree," Entropy, vol. 15, no. 5, pp. 16431662, 2013.

[20] B. Podobnik, Z.-Q. Jiang, W.-X. Zhou, and H. E. Stanley, "Statistical tests for power-law cross-correlated processes," Physical Review E, vol. 84, no. 6, Article ID 066118, 8 pages, 2011.

[21] W.-X. Zhou, "Multifractal detrended cross-correlation analysis for two nonstationary signals," Physical Review E, vol. 77, no. 6, Article ID 066211, 4 pages, 2008.

[22] L. Kristoufek, "Multifractal height cross-correlation analysis: a new method for analyzing long-range cross-correlations," Europhysics Letters, vol. 95, no. 6, Article ID 68001, 6 pages, 2011.

[23] Y. Wang, Y. Wei, and C. Wu, "Detrended fluctuation analysis on spot and futures markets of West Texas Intermediate crude oil," Physica A, vol. 390, no. 5, pp. 864-875, 2011.

[24] G.-J. Wang and C. Xie, "Cross-correlations between WTI crude oil market and U.S. stock market: a perspective from econophysics," Acta Physics Polonica B, vol. 43, no. 10, pp. 20212036, 2012.

[25] E. B. S. Marinho, A. M. Y. R. Sousa, and R. F. S. Andrade, "Using detrended cross-correlation analysis in geophysical data," Physica A, vol. 392, no. 9, pp. 2195-2201, 2013.

[26] X. Zhao, J. Yue, and P. Shang, "Effect of trends on detrended fluctuation analysis of precipitation series," Mathematical Problems in Engineering, vol. 2010, Article ID 749894, 15 pages, 2010.

[27] G. F. Zebende, M. F. da Silva, and A. Machado Filho, "DCCA cross-correlation coefficient differentiation: theoretical and practical approaches," Physica A, vol. 392, no. 8, pp. 1756-1761, 2013.

[28] S. Shadkhoo and G. R. Jafari, "Multifractal detrended crosscorrelation analysis of temporal and spatial seismic data," European Physical Journal B, vol. 72, no. 4, pp. 679-683, 2009.

[29] D. Ghosh, S. Dutta, and S. Samanta, "Fluctuation of gold price: a multifractal approach," Acta Physica Polonica B, vol. 43, no. 6, pp. 1261-1274, 2012.

[30] F. Wen and X. Yang, "Skewness of return distribution and coefficient of risk premium," Journal of Systems Science \& Complexity, vol. 22, no. 3, pp. 360-371, 2009.

[31] P. Gopikrishnan, M. Meyer, L. A. N. Amaral, and H. E. Stanley, "Inverse cubic law for the distribution of stock price variations," European Physical Journal B, vol. 3, no. 2, pp. 139-140, 1998. 
[32] G.-J. Wang and C. Xie, "Cross-correlations between the CSI 300 spot and futures markets," Nonlinear Dynamics, vol. 73, no. 3, pp. 1687-1696, 2013.

[33] F. Wen and Z. Dai, "Modified Yabe-Takano nonlinear conjugate gradient method," Pacific Journal of Optimization, vol. 8, no. 2, pp. 347-360, 2012. 


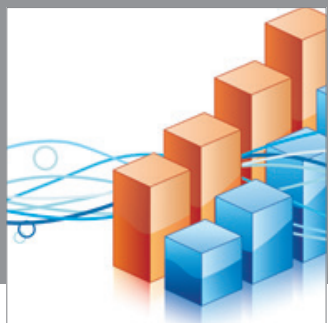

Advances in

Operations Research

mansans

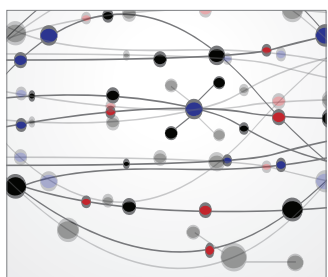

The Scientific World Journal
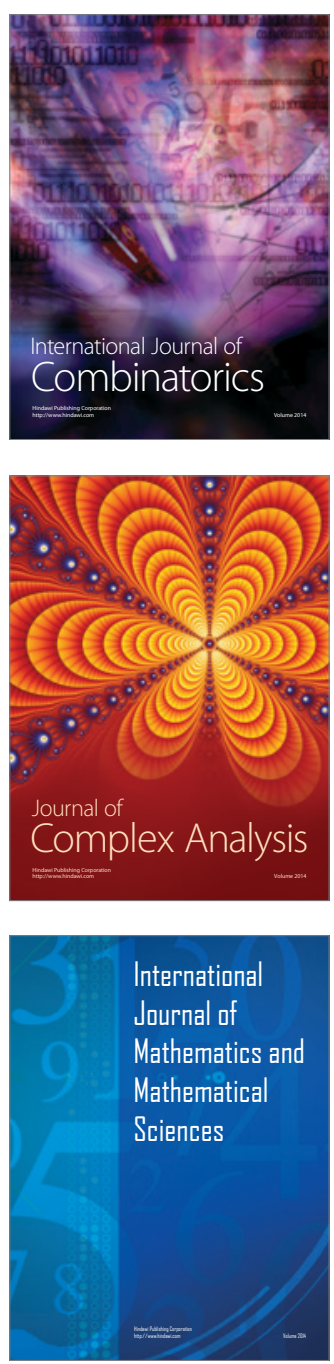
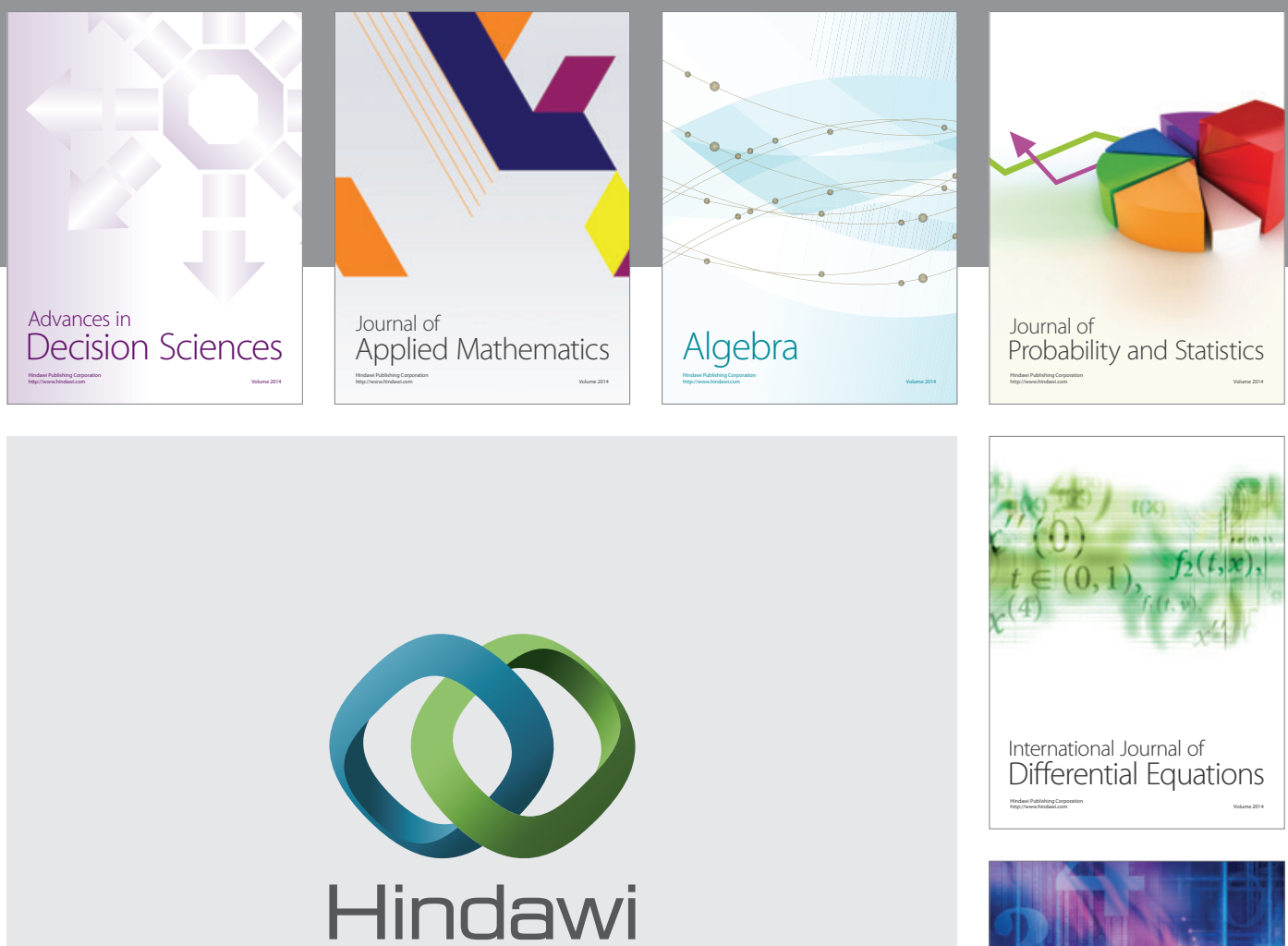

Submit your manuscripts at http://www.hindawi.com
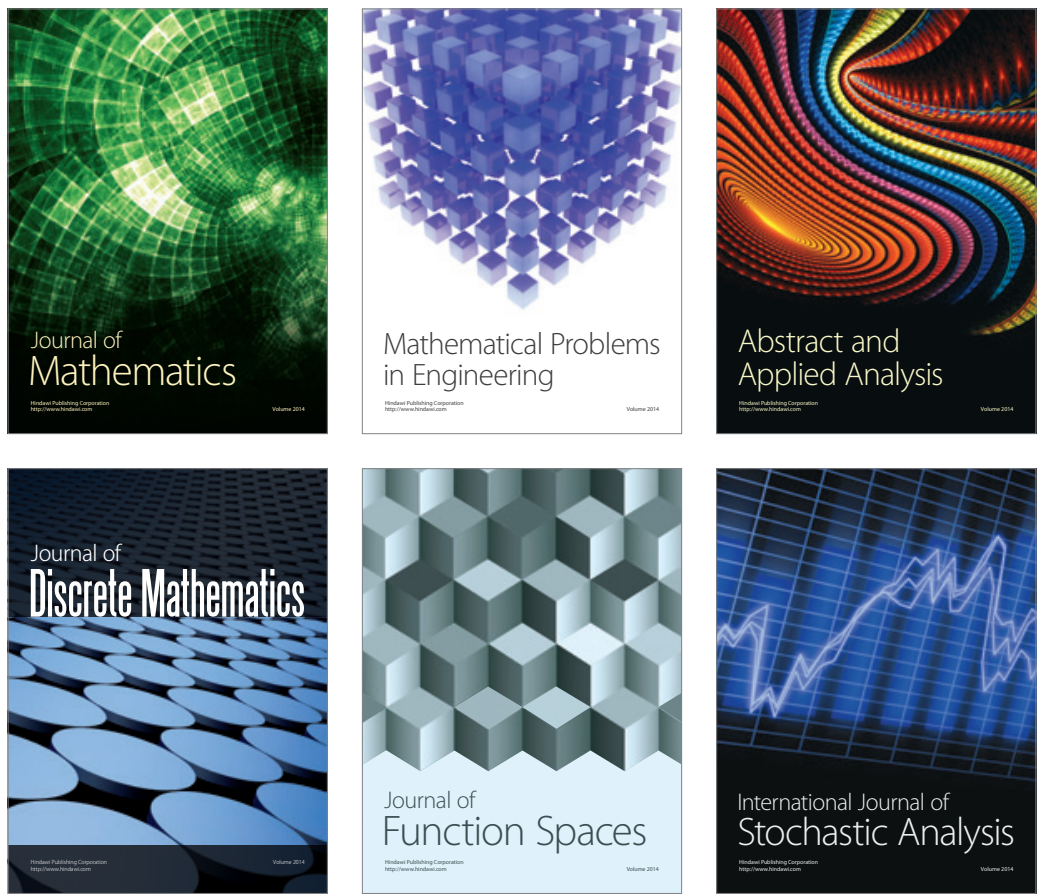

Journal of

Function Spaces

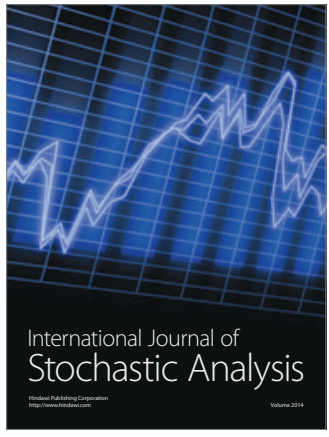

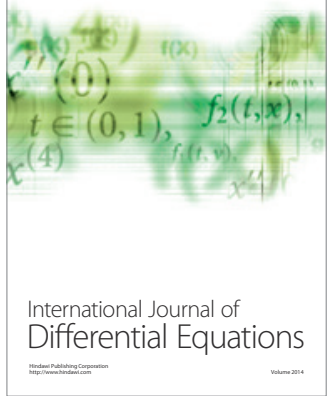
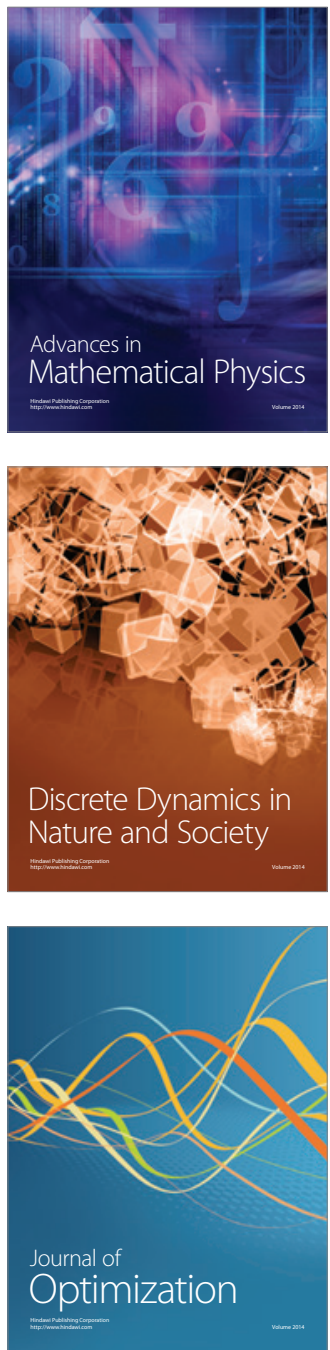